\title{
Micropositioning and microscopic observation of individual picoliter-sized containers within SU-8 microchannels
}

\author{
Martin G. Jenke • Christoph Schreiter • \\ Gyu Man Kim · Horst Vogel · Jürgen Brugger
}

Received: 30 June 2006/ Accepted: 30 August 2006/Published online: 21 September 2006

(C) Springer-Verlag 2006

\begin{abstract}
We describe the fabrication and application of a bioanalytical chip, made of SU-8 photoresist, comprising integrated, high aspect-ratio microfluidic channels, suitable to manipulate and investigate vesicles, cell fragments and biological cells. A central micrometer-sized aperture allows electrical particle counting and planar membrane experiments, microfluids allow (sub)micrometer-sized objects to be transported and addressed with different chemicals. Here we show how lipid vesicles are positioned with micrometer precision within the micro-channels by means of pressure and electrophoretic movement. Our approach is suited for controlling and investigating (bio)chemical synthesis and cellular signalling processes in ultrasmall individual vesicles by electro-optical techniques.
\end{abstract}

Keywords SU-8 $\cdot$ Microfluidics $\cdot$ Electrophoretic micromanipulation $\cdot$ Rapid prototyping

M. G. Jenke · G. M. Kim · J. Brugger $(\bowtie)$

Microsystems Laboratory (LMIS),

Ecole Polytechnique Fédérale de Lausanne (EPFL),

1015 Lausanne, Switzerland

e-mail: juergen.brugger@epfl.ch

M. G. Jenke

e-mail: martin.jenke@epfl.ch

C. Schreiter · H. Vogel ( $\square)$

Laboratory of Physical Chemistry of Polymers

and Membranes (LCPPM),

Ecole Polytechnique Fédérale de Lausanne (EPFL),

1015 Lausanne, Switzerland

e-mail: horst.vogel@epfl.ch

G. M. Kim

School of Mechanical Engineering, Kyungpook National

University, Kyungpook, South Korea

\section{Introduction}

The creation and discovery of a growing number of biological target molecules and potentially therapeutic compounds presently drives bioanalytics to the micro- and nanometer scale in the hope to unravel molecular details of cell function, understand diseases on a genetic basis and discover new medicines in a much more efficient way than by traditional analytical techniques (Juncker and Schmid 2005; Reyes and Iossifidis2002; Auroux and Iossifidis2002; Cooper 2002; Koh and Yau 2003; Jankevics et al. 2005). Of increasing interest in this context are investigations on single cells using laser tweezers (Enger and Goksor 2004; Ozkan and Wang 2003), optical measurement techniques (Durack and Robinson 2000; Kuang and Biran 2004), planar patch clamp technologies on a chip (Schmidt et al. 2000; Klemic and Klemic 2002; Fertig et al. 2002) or the microfluidic probe for the localized addressing of cells (Juncker and Schmid 2005; Pihl and Sinclair 2005). It was recently shown that single cell bioanalytics can be further downscaled to the atto- and femtoliter range by producing native vesicles from live cells and investigating cellular signalling reactions in such individual vesicles (Pick and Schmid 2005). Because about 100 of these (sub-) micrometer sized containers can be produced from a single live cell, single vesicle analytics opens the possibility to multiplex the investigation of biochemical reactions of single cells. On the other hand, there is increasing interest to use individual artificial lipid and polymeric vesicles as containers ideally suited for the isolation, preservation, transport and localization of a few or single molecules. Their ultrasmall dimensions $(>20 \mathrm{~nm})$ al- 
low unparalleled reduction of confined volumes to the zeptolitre range $\left(10^{-21} \mathrm{~L}\right)$ and use them as reaction containers for controlling and investigating (bio)chemical reactions at a novel level of miniaturization (Chiu and Wilson 1999; Karlsson et al. 2001; Stamou et al. 2003; Bolinger and Stamou 2004; Karlsson et al. 2004, 2005; Sukhorukov and Rogach 2005; Graff and Winterhalter 2001). In order to fully exploit the control and monitoring of (bio)chemical synthesis and cellular signalling reactions in single vesicles, methods have to be developed to manipulate and address these containers individually: Artificial lipid vesicles can be produced in microfluidic channels (Anna and Bontoux 2003; Thorsen and Roberts 2001), self-positioned in form of microarrays (Stamou et al. 2003; Jahn and Vreeland 2004; Voros and Blattler 2005), interconnected by a nanoscale channel networks (Karlsson et al. 2004), counted as individuals while passing (sub)micrometer apertures (Schmidt et al. 2000), and spread across micro- and nanometer sized holes for electrophysiological measurements (Schmidt et al. 2000; Fertig et al. 2002; Romer and Steinem 2004).

Here we extend these possibilities by developing a biochip that can be used for simultaneously micromanipulating and investigating optically and electrically individual vesicles in a microfluidic channel. We demonstrate the feasibility of the device by transporting lipid vesicles within the micro-fluidic network by means of pressure control and position them as individuals precisely by electrophoretic movement on a micrometer sized aperture.

\section{Experimental section}

\subsection{Materials and design}

The new chip is designed for micromanipulating vesicles and other (sub-)micrometer sized objects (beads, cell fragments and cells) and for combined optical and electrophysiological measurements. The chip was fabricated using SU-8 as material (Lee and Rishton 1994; Lorenz and Despont 1998), which enables a highly flexible manufacturing of micrometer scale devices. SU-8 is a transparent, negative-tone, epoxy-type photoresist that can be spin-coated and structured in layers that are a few hundred nanometres up to $2 \mathrm{~mm}$ thick. Negative images of an exposure mask can be made in the SU-8 layer by means of standard UV-lithography (365 nm wavelength exposure) and allow outstanding aspect ratios up to $25: 1$. SU-8 is biocompatible and chemically resistant to most organic solvents and many acids (Heuschkel and Guerin 1998). Its surface is negatively charged in aqueous solution, becomes hydrophobic in air but can be re-activated by oxygen plasma and kept hydrophilic in water.

We exploited these characteristics to fabricate a biochip entirely made of SU-8 that consists of a central thin film, a microfluidic channel network and a central $\mu \mathrm{m}$-sized aperture, as shown schematically in Fig. 1. The central aperture can be used to sort vesicles or to position them for subsequent biophysical and bioanalytical investigations, such as fluorescence imaging or electrophysiological measurements (Schmidt et al. 2000; Fertig et al. 2002). The channel configuration enables very convenient liquid handling and micrometer precise positioning of vesicles (and cells). The channel height is given by one of the SU-8 layers and can be varied from 50 to $100 \mu \mathrm{m}$ compatible for microscopic investigations with objectives of high numerical aperture (up to $N A=1.2$ ).

\subsection{Fabrication of microstructured SU-8 chips}

Layers of chrome (Cr, $200 \mathrm{~nm}$ thick) and aluminium (Al, 500-nm-thick) were sequentially deposited by thermal evaporation on a blank silicon $(\mathrm{Si})$ wafer using an Alcatel EVA 600 E-beam/Joule evaporator (Fig. 1, right). The $\mathrm{Cr}$ layer was deposited to improve the adhesion of $\mathrm{Al}$ on the Si surface and to contact the $\mathrm{Al}$ layer during the electrochemical etching. The Al layer, which served as sacrificial layer at a later process step, was structured by dry etching with a STS Multiplex ICP using positive photoresist (Shipley S 1813) as mask, exposed on a standard UV mask aligner (Karl SUSS MA6 Proximity/Contact Double Side Mask Aligner). High-contrast alignment marks where thus obtained in the $\mathrm{Al}$ layer for the subsequent process steps.

After an initial de-hydration bake of $30 \mathrm{~min}$ on a $100^{\circ} \mathrm{C}$ hotplate to remove undesired surface water films and to improve adhesion, several SU-8 layers with different polymeric composition and viscosity where sequentially deposited by spin coating to the glass support. The thickness of the SU-8 layers can be controlled with sub-micrometer precision by adjusting both the viscosity of the SU-8 solution via concentration and the spinning velocity. In our experiment, the different layers consist of "SU-8 50" and "SU-8 70" (Jiguet 2004) that have a SU-8 to solvent ratio of 50/50 and 70/30, respectively. In addition, commercial "SU-8 2035" was used (Microchem, USA). First, a 3.3- $\mu \mathrm{m}$ thick "SU-8 50" layer was spincoated in a two-step spin process $(1,000 \mathrm{rpm}$ for $10 \mathrm{~s}$ and $3,000 \mathrm{rpm}$ remove about $80 \%$ of the solvent. The SU-8 layer was after- 


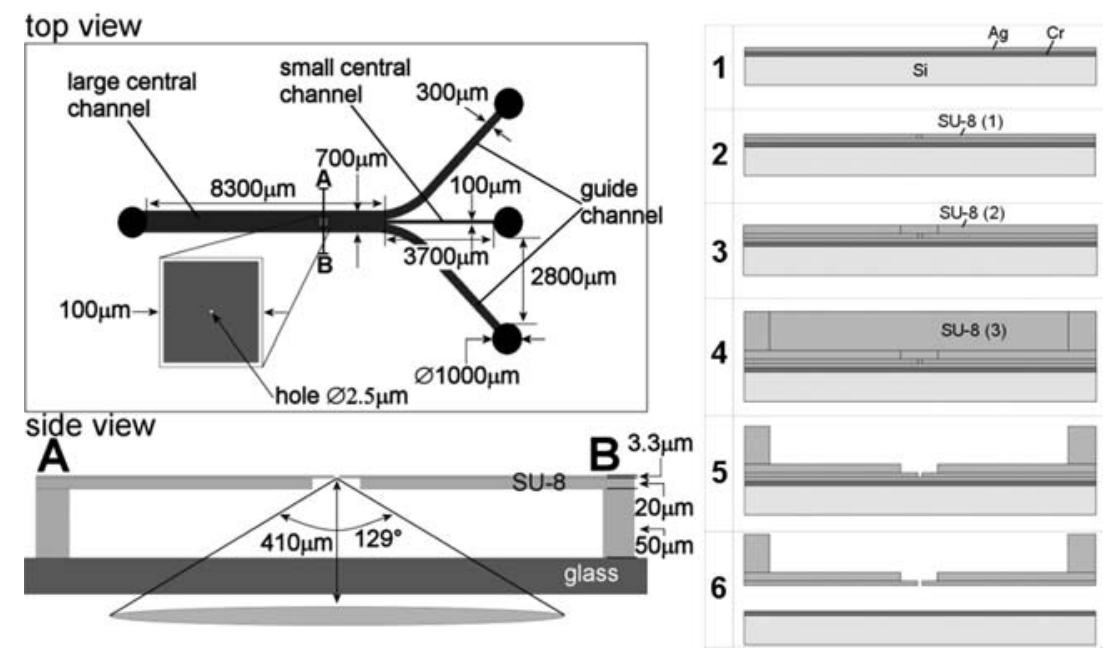

Fig. 1 Schematic view of the SU-8 chip. Left: Top-Top-view of the chip showing the three inlet channels (guide channels and small central channel) convening to form one large central channel. A micrometer-size aperture is fabricated in the large central channel. Bottom-Cross-sectional view of the large central channel at the aperture together with the glass slide that seals the microfludic network and the microscope objective below. Right: Illustrations of the microfabrication process for the

wards exposed to UV light $(365 \mathrm{~nm})$ through a chrome mask $\left(9,16 \mathrm{~mW} / \mathrm{cm}^{2}, 8 \mathrm{~s}\right)$ and then heated at 60 and $90^{\circ} \mathrm{C}$. Without developing the first layer, a second, 20 $\mu \mathrm{m}$-thick layer ("SU-8 70", spin coated at $500 \mathrm{rpm}$ for $5 \mathrm{~s}$ and at 4,000 rpm for $40 \mathrm{~s}, 25 \mathrm{~s}$ exposure) and a third, 50- $\mu$ m-thick SU-8 layer ("SU-8 2035", spin coated at $500 \mathrm{rpm}$ for $5 \mathrm{~s}$ and at 2,200 rpm for $30 \mathrm{~s}$ and $24 \mathrm{~s}$ exposure) were deposited, pre-baked, photopolymerized by UV exposure and post-baked. After the post exposure bake of the last (third) layer, the entire multi-layer structure, consisting of the three exposed SU-8 layers, was simultaneously developed using a SU8 developer (MRT GmbH, Germany).

The 3D structured SU-8 chips were finally released from the wafer by electrochemically etching the sacrificial Al layer applying an electrical potential of $2 \mathrm{~V}$ between the wafer (anode) and an Al foil (cathode) in a saturated $\mathrm{NaCl}$ solution (Datta 1993). To ensure complete opening of the $2.5 \mu \mathrm{m}$ aperture in the first $3.3 \mu \mathrm{m}$ thick SU-8 layer, the chips were placed in the SU-8 developer for additional $8 \mathrm{~min}$.

\subsection{Experimental set-up}

The microfabricated and released biochips were mounted 'sandwich-like' on a $170 \mu \mathrm{m}$ thick standard microscope slide, sealed by a PDMS pad, and fixed with a PMMA plate that was screwed to the microscope holder (Fig. 2). The PDMS pad served as sealing integrated, planar SU-8 chip. (1) Vacuum deposition of a 200nm-thick Chrome and a 500-nm-thick Aluminium sacrificial layer by evaporation on polished silicon wafer. (2-4) Spin coating, prebake, exposure and post exposure bake of three consecutive SU-8 layers, 3.3/20/50 $\mu \mathrm{m}$ thick, respectively. (5) Development of the exposed triple SU-8 layer. (6) Surface releasing by electrochemical etching of Aluminium

material and spacer, and provided access holes for the fluids and the electrodes. Holes in the PMMA plate allowed the fluidic connection to a pump comprising 10-25 $\mu \mathrm{l}$ syringes for automatic fluidic delivery and pressure control.

The chip was placed at an inverted fluorescence microscope (Zeiss, Germany) equipped with a $\mathrm{Hg} / \mathrm{Xe}$ lamp and adequate filters for exciting and observing rhodamine fluorescence (excitation $545 \mathrm{~nm}$, emission $570 \mathrm{~nm}$ ). Images were taken with a FAC $830 \mathrm{CCD}$ camera (Grundig, Germany) in phase contrast using $10 \times$ and $40 \times$ Zeiss objectives.

\subsection{Vesicles, buffers}

Giant unilamellar vesicles were prepared by overnight swelling dried lipid films of $80 \%$ 1,2-dioleoyl-sn-glycero-3-phosphatidylcholine (DOPC), 20\% 1-palmitoyl2-oleoyl-sn-glycero-3-phosphatidylglycerol (POPG) (Avanti Polar Lipids, USA) in $10 \mathrm{ml}$ of $200 \mathrm{mM}$ sorbitol solution. For fluorescence detection, lipid vesicles were labelled with $0.5 \%$ of $\mathrm{N}$-(6-tetramethylrhodaminethiocarbamoyl)-1,2-dihexadecanoyl-sn-glycero-3phosphatidyl-ethanolamine (TRITC-DHPE) (Molecular Probes, USA). Giant unilamellar vesicles had typical sizes up to $60 \mu \mathrm{m}$ diameter and were stored at $4{ }^{\circ} \mathrm{C}$. All chemicals were from regular sources and of highest chemical grade. 


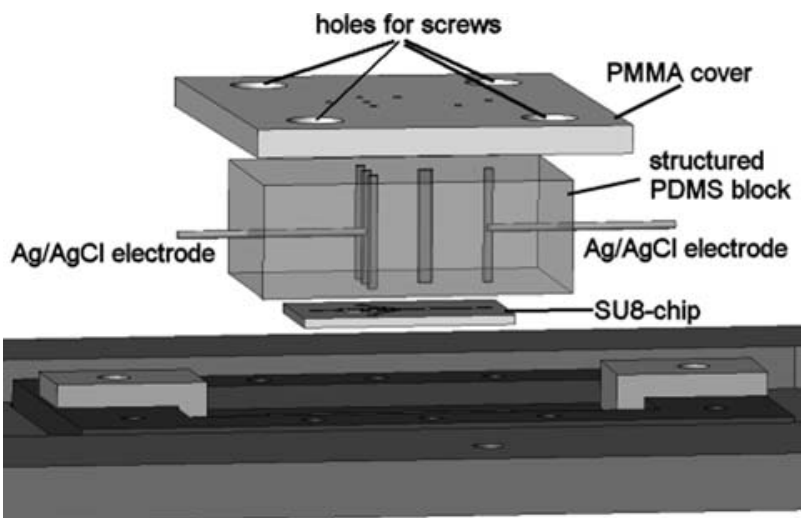

support with microscope glass slide

Fig. 2 Mounting of the SU-8 chip on the microscope. The SU-8 chip is fixed in a stack of glass, PMMA and PDMS. The PDMS block serves as sealing material and provides access holes for the buffer solutions to the microchannels and to the top-side of the chip. Two $\mathrm{Ag} / \mathrm{AgCl}$ electrodes mounted in the PDMS block are in contact with the electrolyte solution of the central entrance and the exit channel. A third electrode (not shown for simplicity) is connected to the top-side of the chip enabling electrophoretic positioning. The PMMA cover is screwed to the microscope stage and provides access holes to connect tubings to the microchannels

\section{Results and discussion}

The fabricated chip consists of three inlet channels and one outlet channel. The design of the chip (Fig. 1) and the laminar flow characteristics allows the middle inlet channel flow to transport the vesicles or chemical compounds under study directly over the fabricated central hole. The scanning electron microscope (SEM) images in Fig. 3 show parts of the microchannels and the hole. The geometry of the channel is well defined and the SU-8 film with the hole is clearly visible. The diameter of the hole in the chrome mask for lithography is $5 \mu \mathrm{m}$, the diameter of the hole in the SU-8 film at the end of the lithographic process is about $2.5 \mu \mathrm{m}$. The reduction of the hole in the SU-8 film is due to the multiple SU-8 layer processes (exposure and baking steps).

The behaviour of the micro flow in the chip was observed by light transmission and fluorescence microscopy, injecting water into the central channel and an aqueous solution of rhodamine into the neighbouring guide channels. Figure 4 shows the confluence of the three channels with three clearly separated laminar flows. The same flow behaviour was observed over the aperture.

By varying the inlet flow rate and pressure of each of the three channels, it was possible to modulate the width of the central flow between 20 and $80 \mu \mathrm{m}$, and to laterally displace the central flow by up to $300 \mu \mathrm{m}$ from

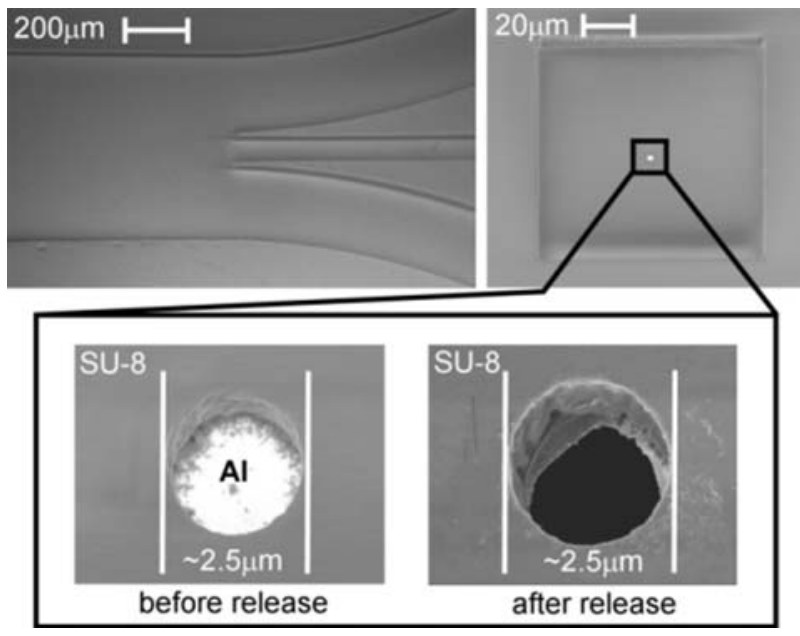

Fig. 3 Scanning electron microscope (SEM) image of the chip. a Merger of the three inlet micro-channels to the large central channel. b SU-8 membrane with central hole. c Enlarged view of the hole before releasing (the aluminium layer is still visible). d Same as (c) but after release of SU-8 chip yielding a $2.5 \mu \mathrm{m}$ hole in a thin SU-8 membrane
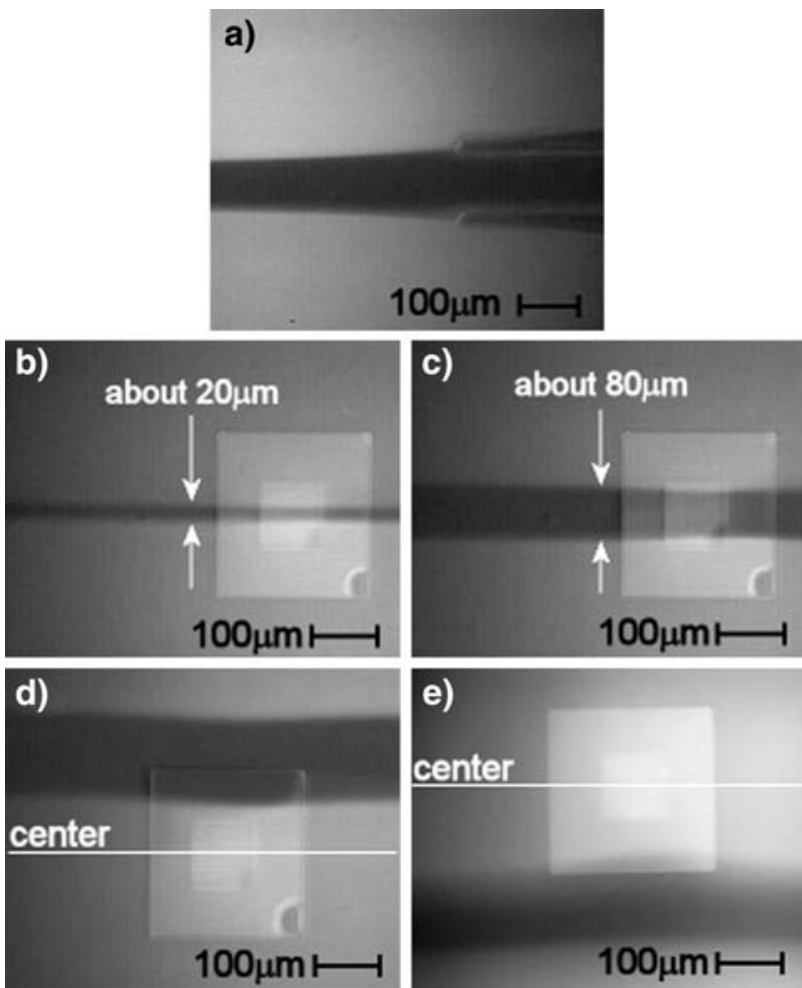

Fig. 4 Optical microscope images of the three confluent laminar flows. a Inlet of the three micro-channels into the large central channel. The dark $\mathrm{H}_{2} \mathrm{O}$ flow leaves the small central channel. The two coloured aqueous/rhodamin solution adjacent flows leave the guide channels. The width of the central flow is controlled by adjusting the pressure of the two surrounding flows. b $20 \mu \mathrm{m}$-wide central laminar flow c $80 \mu \mathrm{m}$-wide central laminar flow. d, e Lateral displacement of the central laminar flow by varying the neighbouring channel flow and pressure 
the central axis of the chip. The flow speed could be varied from 0.1 to $50 \mathrm{~mm} / \mathrm{s}$ without changing the flow characteristics of the system. In this way, the microfluidic system could be used as a perfusion system to rapidly apply chemical compounds such as ligands to a target vesicle trapped on the hole.

The same flow principle was applied to guide vesicles at low speed $(0.1 \mathrm{~mm} / \mathrm{s})$ towards the membrane and the hole. Fine positioning onto the hole was done by electrophoretic movement. Three chlorinated silver rods integrated in the PDMS block were used as electrodes (Fig. 2). The laminar flow was stopped when a suitable vesicle arrived near the central SU-8 membrane with hole and a voltage between 10 and $15 \mathrm{~V}$ was then applied to position with micrometer precision a vesicle to the central hole (Fig. 5). Upon contact with the chip surface, the vesicle opens and spreads forming a planar lipid bilayer spanning the aperture (Danelon et al. 2006).

Taken together, we fabricated by a simple and highly flexible photolithography processes a 3D structured all-SU-8 chip, which is fully transparent, biocompatible (due to the SU-8 material characteristics) and has a microfluidic network connected to a central micrometer-sized aperture. A dedicated microscope holder provided the fluidic and electrical connections to the chip. The flow speed could be varied from $250 \mathrm{nl} / \mathrm{min}$ to hundreds of $\mu \mathrm{l} / \mathrm{min}$ without changing the flow characteristics of the system. Single lipid vesicles were transported and positioned with micrometer precision in the microchannel on the central hole using a combination of pressure flow and electrophoretic movement.
In our present communication we concentrated on the use of lipid vesicles. They can be optimally designed as tight and inert containers for performing many interesting chemical and biochemical processes. The potential of these systems for miniaturization and bionanotechnology was nevertheless realized only after single vesicles were extracted from the ensemble and addressed as individuals, either by means of micromanipulation or by directed assembly on patterned surfaces (Stamou et al. 2003).

Our results on the transport and micromanipulation of vesicles in microchannels open the door for a number of novel bioanalytical applications both for artificial or native cell-derived vesicles: (1) Vesicles are ideal vehicles to transport few or single molecules (Bolinger and Stamou 2004; Karlsson et al. 2004, 2005; Sukhorukov and Rogach 2005; Graff and Winterhalter 2001) and highly complex functionalities (Pick and Schmid 2005) in defined ultrasmall volumes over macroscopic distances without loss, and finally position them with micrometer precision at solid supports (Stamou et al. 2003; Voros and Blattler 2005). (2) Because individual vesicles can be precisely and quickly moved between laminar flows of varying chemical composition within a microchannel (or vice versa, the different laminar flows can be rapidly displaced across on immobilized vesicles), it is possible to induce fast chemical mixing experiments, comparable to classical stopped-flow experiments, but here at ultrasmall volumes down to zeptoliters at the miniaturized biochip with simultaneous optical or electrical detection and single molecule resolution. (3) The micrometer precise movement of the vesicles opens a
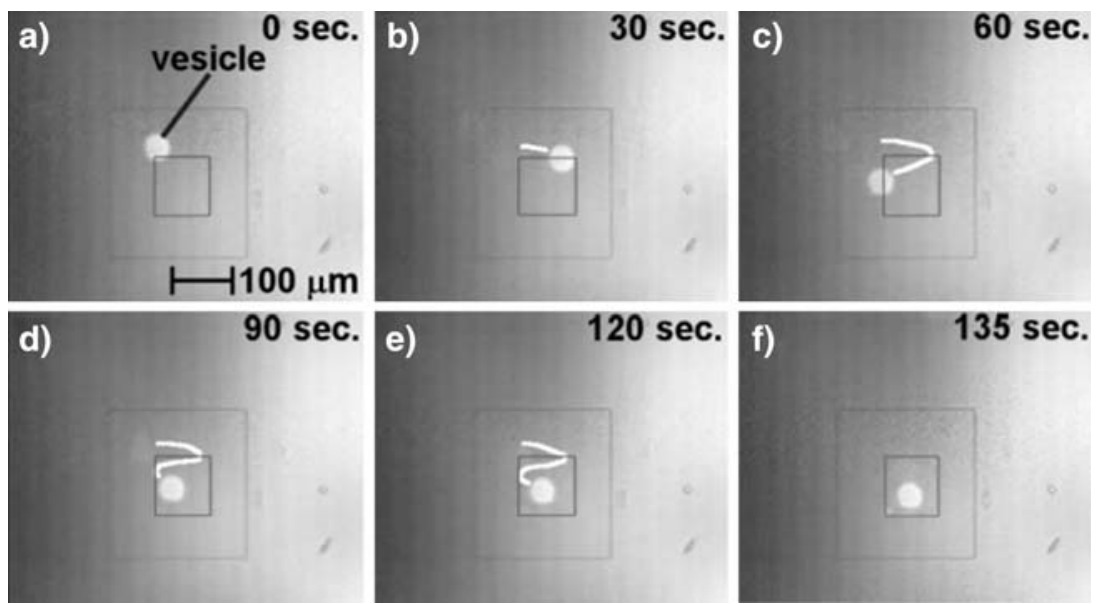

Fig. 5 Directing a negatively charged lipid vesicle towards the central hole by applying an electric potential of $10 \mathrm{~V}$ between top and bottom electrodes. Images a-e Trajectory (white line drawn artificially) of the vesicle during its passage towards the central hole within a time period of $2 \mathrm{~min}$. Image $\mathbf{f}$ shows the vesicle after $135 \mathrm{~s}$ on the aperture (not visible on this photograph due to resolution limit). The vesicle showed only small oscillating movements on the hole, due to the movement of the surrounding water. (The $3.3 \mu \mathrm{m}$ thick SU-8 film is highlighted by a rectangle) 
number of interesting possibilities for positioning and sorting few or single molecules and "biological" functionalities on solid supports in form of microarrays or across micro-/nanometer-sized apertures either in form of vesicles or after vesicle spreading as planar membranes for performing controlled (bio)chemical synthesis and investigate by optical and electrical detection complex biochemical reactions at ultrasmall dimensions in a highly parallel format.

Acknowledgments We thank Sébastien Jiguet for his remarks and help with the SU-8 process, the CMI staff for the technical assistance, the TopNano21 programme (HV) and the EPFL (JB and HV) for financial support.

\section{References}

Anna SL, Bontoux N, Stone HA (2003) Formation of dispersions using "flow focusing" in microchannels. Appl Phys Lett 82(3):364-366

Auroux PA et al (2002) Micro total analysis systems. 2. Analytical standard operations and applications. Anal Chem 74(12):2637-2652

Bolinger PY, Stamou D, Vogel H (2004) Integrated nanoreactor systems: triggering the release and mixing of compounds inside single vesicles. J Am Chem Soc 126(28):8594-8595

Chiu DT et al (1999) Chemical transformations in individual ultrasmall biomimetic containers. Science 283(5409):18921895

Cooper MA (2002) Optical biosensors in drug discovery. Nat Rev Drug Discov 1(7):515-528

Danelon C, Jenke MG, Schreiter C, Kim GM, Perez JB, Santschi C, Brugger J, Vogel H (2006) Micro- and Nanostructured Devices for Investigating Biomolecular Interactions. Chimia (in press)

Datta M (1993) Anodic-dissolution of metals at high-rates. Ibm J Res Dev 37(2):207-226

Durack G, Robinson JP (2000) Emerging tools for single-cell analysis. Wiley-Liss, New York

Enger $\mathbf{J}$ et al (2004) Optical tweezers applied to a microfluidic system. Lab Chip 4(3):196-200

Fertig N, Blick RH, Behrends JC (2002) Whole cell patch clamp recording performed on a planar glass chip. Biophys $\mathrm{J}$ 82(6):3056-3062

Graff A, Winterhalter M, Meier W (2001) Nanoreactors from polymer-stabilized liposomes. Langmuir 17(3):919-923

Heuschkel MO et al (1998) Buried microchannels in photopolymer for delivering of solutions to neurons in a network. Sens Actuators B Chem 48(1-3):356-361

Jahn A et al (2004) Controlled vesicle self-assembly in microfluidic channels with hydrodynamic focusing. J Am Chem Soc 126(9):2674-2675

Jankevics H et al (2005) Diffusion-time distribution analysis reveals characteristic ligand-dependent interaction patterns of nuclear receptors in living cells. Biochemistry 44(35):11676-11683

Jiguet $\mathrm{S}$ et al (2004) SU8-silver photosensitive nanocomposite. Adv Eng Mater 6(9):719-724

Juncker D, Schmid H, Delamarche E (2005) Multipurpose microfluidic probe. Nat Mater 4(8):622-628

Karlsson A et al (2001) Molecular engineering - networks of nanotubes and containers. Nature 409(6817):150-152

Karlsson M et al (2004) Biomimetic nanoscale reactors and networks. Annu Rev Phys Chem 55:613-649

Karlsson A et al (2005) Controlled initiation of enzymatic reactions in micrometer-sized biomimetic compartments. J Phys Chem B 109(4):1609-1617

Klemic KG et al (2002) Micromolded PDMS planar electrode allows patch clamp electrical recordings from cells. Biosens Bioelectron 17(6-7):597-604

Koh HL et al (2003) Current trends in modern pharmaceutical analysis for drug discovery. Drug Discov Today 8(19):889_ 897

Kuang Y, Biran I, Walt DR (2004) Simultaneously monitoring gene expression kinetics and genetic noise in single cells by optical well arrays. Anal Chem 76(21):6282-6286

Lee KY, Rishton SA, Chang THP (1994) High-aspect-ratio aligned multilayer microstructure fabrication. J Vac Sci Technol B 12(6):3425-3430

Lorenz H et al (1998) High-aspect-ratio, ultrathick, negativetone near-UV photoresist and its applications for MEMS. Sens Actuators A Phys 64(1):33-39

Ozkan M et al (2003) Optical manipulation of objects and biological cells in microfluidic devices. Biomed Microdevices 5(1):61-67

Pick $\mathrm{H}$ et al (2005) Investigating cellular signaling reactions in single attoliter vesicles. J Am Chem Soc 127(9):2908-2912

Pihl J et al (2005) Microfluidic gradient-generating device for pharmacological profiling. Anal Chem 77(13):3897-3903

Reyes DR et al (2002) Micro total analysis systems. 1. Introduction, theory, and technology. Anal Chem 74(12):2623-2636

Romer W, Steinem C (2004) Impedance analysis and singlechannel recordings on nano-black lipid membranes based on porous alumina. Biophys J 86(2):955-965

Schmidt C, Mayer M, Vogel H (2000) A chip-based biosensor for the functional analysis of single ion channels. Angew Chem Int Ed 39(17):3137-3140

Stamou D et al (2003) Self-assembled microarrays of attoliter molecular vessels. Angew Chem Int Ed 42(45):5580-5583

Sukhorukov GB et al (2005) Nanoengineered polymer capsules: tools for detection, controlled delivery, and site-specific manipulation. Small 1(2):194-200

Thorsen T et al (2001) Dynamic pattern formation in a vesiclegenerating microfluidic device. Phys Rev Lett 86(18):41634166

Voros J, Blattler T, Textor M (2005) Bioactive patterns at the $100-\mathrm{nm}$ scale produced using multifunctional physisorbed monolayers. MRS Bull 30(3):202-206 